\title{
Evolution by flight and fight: diverse mechanisms of adaptation by actively motile microbes
}

\author{
Olaya Rendueles and Gregory J Velicer \\ Institute for Integrative Biology, ETH Zürich, Universitätstrasse 16, Zürich, Switzerland
}

\begin{abstract}
Evolutionary adaptation can be achieved by mechanisms accessible to all organisms, including faster growth and interference competition, but self-generated motility offers additional possibilities. We tested whether 55 populations of the bacterium Myxococcus xanthus that underwent selection for increased fitness at the leading edge of swarming colonies adapted by swarming faster toward unused resources or by other means. Populations adapted greatly but diversified markedly in both swarming phenotypes and apparent mechanisms of adaptation. Intriguingly, although many adapted populations swarm intrinsically faster than their ancestors, numerous others do not. Some populations evolved interference competition toward their ancestors, whereas others gained the ability to facultatively increase swarming rate specifically upon direct interaction with ancestral competitors. Our results both highlight the diverse range of mechanisms by which actively motile organisms can adapt evolutionarily and help to explain the high levels of swarming-phenotype diversity found in local soil populations of $M$. xanthus.

The ISME Journal (2017) 11, 555-568; doi:10.1038/ismej.2016.115; published online 23 September 2016
\end{abstract}

\section{Introduction}

A myriad of adaptations promoting migration have evolved (Swingland and Greenwood, 1983), including common behaviors such as walking and swimming and more unusual behaviors such as ballooning (Reynolds et al., 2006) and phoresy (Magsig-Castillo et al., 2010). In particular, self-generated motility is a common mode of evolutionary adaptation across all domains of life and greatly affects the spatial distributions of organisms (Bowler and Benton, 2005). Self-propelled movement allows, among other benefits, active foraging (Stocker et al., 2008; Taylor and Stocker, 2012), means to escape dangerous circumstances (Taylor and Buckling, 2013) and enhanced dispersal to and colonization of new habitats (Ottemann and Miller, 1997), all of which can be highly beneficial in changing environments. In non-motile species, evolutionary adaptation can occur by increasing the maximum rate of reproduction in any given environment (Travisano et al., 1995), exploiting novel resource niches (Blount et al., 2008) or evolving novel modes of interference competition (Lemonnier et al., 2008). In contrast, motile organisms have the additional potential to adapt by modifying the rate, pattern or energetics of their locomotive behaviors.

Correspondence: O Rendueles Genomes \& Genetics, Institut Pasteur, 28 r Dr Roux, Paris 75724 France.

E-mail: olaya.rendueles-garcia@pasteur.fr

Received 21 January 2016; revised 19 June 2016; accepted 3 July 2016; published online 23 September 2016
Among microbes, motility is essential for many individual- and group-level behaviors, including migration toward nutrient sources (Taylor and Buckling, 2011; van Ditmarsch et al., 2013), host colonization (Turnbull et al., 2001; Josenhans and Suerbaum, 2002), biofilm and fruiting-body formation (Pratt and Kolter, 1998; Velicer and Vos, 2009) and predation (Velicer and Vos, 2009). Owing to their rapid growth, large populations and genetic tractability, microbial systems allow evolution experiments to examine the roles of motility in adaptation in ways that are not feasible with large organisms (Kawecki et al., 2012). One evolution experiment with the soil-dwelling cooperative bacterium Myхососcus xanthus showed that bacterial motility can rapidly degrade under relaxed selection (Velicer et al., 1998). In contrast, motility rate can increase evolutionarily during adaptation in a spatially structured habitat in which resource patches are relatively rare (Hillesland and Velicer, 2005). In three other experimental evolution studies (one with $M$. xanthus and two with Pseudomonas aeruginosa), repeated exclusive selection of cells at the leading edge of expanding colonies for transfer to the next evolutionary cycle universally led to increased rates of swarming in evolved populations (Velicer and Yu, 2003; Taylor and Buckling, 2011; van Ditmarsch et al., 2013).

$M$. xanthus uses two genetically and functionally different motility systems to search for prey (Hodgkin and Kaiser, 1979; Shi and Zusman, 1993). 'S motility', also known as 'Type IV pili-mediated motility' (Sun et al., 2000) is a group-coordinated 
mode of movement that requires cell-cell proximity and is driven by Type IV pili (Kaiser, 1979; Wu and Kaiser, 1995). Adventurous motility ('A motility') allows cells to move individually and involves transient focal adhesion complexes (Mignot et al., 2007; Sun et al., 2011). These motility systems contribute to group movement to different degrees on different surfaces (Hodgkin and Kaiser, 1979; Shi and Zusman, 1993) and each is genetically complex (Youderian et al., 2003; Youderian and Hartzell, 2006). Swarming on soft (0.5\%) agar is driven solely by $\mathrm{S}-$ motility, as $\mathrm{A}+\mathrm{S}-$ mutants are unable to swarm effectively on soft agar but A-motility mutants $(\mathrm{A}-\mathrm{S}+$ ) swarm equivalently to wild-type $\mathrm{A}+\mathrm{S}+$. In contrast, both motility systems contribute to swarming on hard $(1.5 \%)$ agar, as debilitation of either system reduces but does not eliminate swarming on hard agar (Shi and Zusman, 1993). Such dual motility is thought to allow $M$. xanthus to swarm more effectively across diverse surface conditions in the soil (Young and Crawford, 2004) than would either system alone (Shi and Zusman, 1993). Motility rates among closely related natural isolates of $M$. xanthus isolated from the same local population vary greatly (Vos and Velicer, 2008; Kraemer and Velicer, 2011), indicating (along with the large changes in motility rates and phenotypes previously documented in laboratory populations (Velicer and Yu, 2003; Hillesland and Velicer, 2005)) that bacterial motility evolves rapidly, whether in response to direct selection on motility behavior per se or indirectly by pleiotropy.

In this study, we have tested more extensively than previous studies whether adaptation by actively motile bacteria under continuous selection to increase fitness at the leading edge of expanding swarms occurs solely or predominantly by the evolution of faster swarming, or rather might commonly occur by mechanisms accessible to nonmotile organisms as well. We further tested whether evolutionary changes in motility behavior occurred only by mere changes in intrinsic motility rate or whether populations evolved motility behaviors that are exhibited only in the presence of competitors. Finally, our experimental design, which employed three ancestral motility genotypes evolving in replicate populations across two environments, allowed us to investigate whether the motility genotype of ancestors and/or swarming environment influenced the direction, degree and diversity of phenotypic evolution across several traits related to fitness.

Replicate populations of $M$. xanthus were established from each of three ancestral motility genotypes: A+S+ (both motility systems intact), A-S+ (non-functional A motility) or $\mathrm{A}+\mathrm{S}-$ (non-functional S motility). The two motility systems were debilitated in the respective mutant ancestors by clean deletion of either $\operatorname{cglB}(\mathrm{A}-\mathrm{S}+)$ or pilA $(\mathrm{A}+\mathrm{S}-)$ (see Supplementary Methods), which encode proteins essential for the function of A- and S-motility, respectively (Wu and Kaiser, 1997; Rodriguez and
Spormann, 1999). Populations were repeatedly allowed to grow as outwardly swarming colonies on both hard and soft agar surfaces (Velicer and Yu, 2003; Rendueles et al., 2015) (Supplementary Table S1, Supplementary Figure S1). Parallel evolving populations were initiated from independently isolated subclones of both rifampicin sensitive and resistant variants of each of the three ancestral motility genotypes (Supplementary Figure S1). Every 2 weeks, a population sample from the most distal point of each expanding swarm (or from a random location on the leading edge for circular swarms) was transferred to the center of a new plate and this process was repeated for 40 cycles. Subsequently, we tested evolved populations for (i) adaptation relative to the ancestor in mixed competitions during active swarming, (ii) potential mechanisms of adaptation, either by changes in motility-independent growth rate, changes in pure-culture motility rate, motilityindependent interference competition and effects of competition on motility behavior and (iii) genotypic and environmental effects on the degree and patterns of evolutionary change at both phenotypic and genotypic levels.

\section{Results}

Relative fitness increased in all populations

To demonstrate that evolutionary adaptation occurred, we tested whether the ancestral strains would lose direct competitions while swarming when mixed pairwise with evolved populations. A sample of each evolved population was mixed at a 1:1 initial ratio with a variant of its respective ancestor marked with kanamycin resistance and the resulting chimeric colony was allowed to swarm outward for 7 days on the surface type on which the descendant population had evolved. We then tested whether the evolved population had gone to fixation at the leading edge of the initially mixed colony by harvesting multiple samples from the colony perimeter. $M$. xanthus cells strongly cohere during growth on agar plates to a degree not readily overcome by experimental disaggregation methods, which prevented the use of traditional dilution plating techniques to quantify competitor frequencies. We therefore tested for adaptation by scoring the simple presence or absence of the ancestor at the swarm edge. Complete loss of the ancestor from the leading edge during the competition implies that the fitness of evolved populations increased greatly.

Ancestral cells were completely absent from the swarm-edge region in $78 \%$ of competition pairs (43/55) (one evolved population, P47, was not analyzed), clearly demonstrating the large fitness superiority of these evolved populations (Table 1 and Supplementary Table S1). Moreover, in the 12 competitions in which ancestral cells were present at the edge of competition swarms in at least one sample of one replicate, observational evidence 
Table 1 Summary of significant changes in potentially adaptive traits across all evolved populations

\begin{tabular}{|c|c|c|c|c|c|c|c|}
\hline & Adapted ${ }^{a}$ & Pure swarm speed ${ }^{b}$ & Growth rate ${ }^{c}$ & Growth interference ${ }^{d}$ & Mixed swarm speed ${ }^{e}$ & \# sign. changes ${ }^{f}$ & frz $m u t^{g}$ \\
\hline \multicolumn{8}{|c|}{$A+S+H A$} \\
\hline P1 & +++ & & & & & 0 & $F$ \\
\hline $\mathrm{P} 2$ & +++ & & + & & + & 2 & $C D$ \\
\hline P3 & +++ & & & & & 0 & $F$ \\
\hline $\mathrm{P} 4$ & + & + & & & & 1 & $Z$ \\
\hline P5 & ++ & + & & & & 1 & $F$ \\
\hline P6 & +++ & & & & & 0 & $E$ \\
\hline P7 & +++ & & & & & 1 & $C D$ \\
\hline P8 & +++ & + & & + & & 1 & $F$ \\
\hline P9 & +++ & & & & & 0 & $F / F$ \\
\hline P10 & +++ & & & & & 0 & $F$ \\
\hline P11 & +++ & - & & & & 0 & $F$ \\
\hline P12 & +++ & & & & & 0 & $E / C D$ \\
\hline \multicolumn{8}{|c|}{$A-S+H A$} \\
\hline P13 & +++ & + & & + & & 2 & - \\
\hline P14 & +++ & + & & & & 1 & - \\
\hline P15 & +++ & + & + & & & 2 & - \\
\hline P16 & +++ & + & & & & 1 & - \\
\hline P17 & +++ & + & & & & 1 & - \\
\hline P18 & +++ & + & & & + & 2 & - \\
\hline P19 & +++ & + & & & & 1 & - \\
\hline P20 & +++ & + & & & & 1 & - \\
\hline \multicolumn{8}{|c|}{$A+S-H A$} \\
\hline P21 & ++ & + & & & & 1 & $C D$ \\
\hline P22 & +++ & + & & & & 1 & $E$ \\
\hline P23 & ++ & + & & & & 1 & $F$ \\
\hline P24 & ++ & + & & & & 1 & $F$ \\
\hline P25 & +++ & + & & & + & 2 & $C D$ \\
\hline P26 & +++ & + & & & & 1 & - \\
\hline P27 & +++ & + & & & & 1 & - \\
\hline $\mathrm{P} 28$ & ++ & + & + & & & 2 & $F$ \\
\hline \multicolumn{8}{|c|}{$A+S+S A$} \\
\hline P29 & +++ & + & & & & 1 & $F / C D$ \\
\hline P30 & +++ & + & + & & & 2 & $F$ \\
\hline P31 & +++ & & & & + & 1 & $F$ \\
\hline P32 & +++ & + & + & & + & 3 & $F$ \\
\hline P33 & +++ & & & & & 0 & $F$ \\
\hline P34 & +++ & + & & & & 1 & - \\
\hline P35 & + & & & + & & 0 & $F$ \\
\hline P36 & +++ & & + & & & 1 & $E$ \\
\hline P37 & +++ & & & & & 1 & $F$ \\
\hline P38 & +++ & + & + & & + & 3 & $F$ \\
\hline P39 & +++ & & & & & 0 & $F$ \\
\hline $\mathrm{P} 40$ & +++ & - & + & & & 1 & $F$ \\
\hline \multicolumn{8}{|c|}{$A-S+S A$} \\
\hline P41 & +++ & - & + & & & 0 & $F$ \\
\hline P42 & +++ & + & + & & & 2 & - \\
\hline P43 & +++ & + & + & & & 2 & $F$ \\
\hline P44 & +++ & & & & & 0 & $F$ \\
\hline P45 & +++ & + & & & - & 1 & $F$ \\
\hline P46 & +++ & & & & & 0 & $E$ \\
\hline $\mathrm{P} 48$ & ++ & & & & & 0 & - \\
\hline \multicolumn{8}{|c|}{$A+S-S A$} \\
\hline P49 & +++ & & & + & & 1 & $F$ \\
\hline P50 & +++ & + & & & & 1 & $F$ \\
\hline P51 & +++ & + & & & & 1 & $E$ \\
\hline P52 & + & & & & & 0 & $E$ \\
\hline P53 & + & + & & + & & 2 & $E / Z$ \\
\hline P54 & ++ & & & & & 0 & $Z / C D$ \\
\hline P55 & ++ & + & & + & & 2 & $E$ \\
\hline P56 & +++ & + & & & & 1 & $F$ \\
\hline
\end{tabular}

Abbreviations: HA, hard agar; SA, soft agar.

${ }^{\mathrm{a}}+++$ complete exclusion of ancestor from edge, ++ ancestor present in fewer than $50 \%$ of edge samples, + ancestor present in more than $50 \%$ of the edge samples but at reduced frequency relative to controls.

bignificant changes in pure-culture swarming rates compared with ancestor.

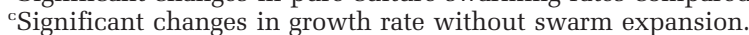

${ }^{\mathrm{d}}$ Significant reduction of ancestor growth in non-swarming competitions.

eSignificantly increased swarming rate in the presence of the ancestor. ${ }^{\text {b-e }}$ Plus $(+)$ symbols indicate statistically significant $(P<0.05)$ evolutionary changes in the respective traits.

${ }^{\mathrm{f}}$ Total number of significant trait changes.

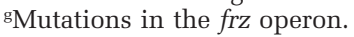


clearly indicated that they constituted only a minority of the sampled population. After control competitions between marked vs unmarked ancestors (see Supplementary Material), robust growth of the marked ancestor was always evident within 2 days after samples from the competition plates were transferred to agar plates containing antibiotic to which the marked ancestor was resistant. In contrast, growth of ancestors transferred from competitions against evolved populations was never observable after 2 days, but only after at least three days, indicating that ancestor population sizes were smaller after growth in the presence of evolved strains than after growth with a differentially marked ancestor. Thus, as expected, all 55 populations showed clear evidence of large gains in fitness relative to their ancestor in the environment in which they evolved.

\section{Mechanisms of adaptation}

Although all populations increased in relative fitness, replicate populations from the same evolutionary treatment were observed to have diversified extensively in several colony phenotypes, including swarming rate (examined more extensively below), morphology and color (Figure 1, Supplementary Figure S2 and S3 and Supplementary Material). This phenotypic diversity suggested that replicate populations had evolved by a diverse range of mechanisms (or combinations of multiple mechanisms). In our selection regime, populations might have adapted by several possible mechanisms: (i) increasing their intrinsic swarming rate, (ii) increasing their intrinsic growth rate independently of swarming rate, (iii) evolving the ability to facultatively increase swarming rate in response to the presence of competitors and (iv) evolving interference competition (that is, the ability to directly hinder the growth or swarming of competitors). We tested for evidence of each of these mechanisms in all 55 populations.

Most evolved populations swarm faster than their ancestor, but many do not. Individual swarming rates of 30 evolved populations were significantly higher than those of their respective ancestors (Figures 2a and b, Table 1 and Supplementary Table S3). The number of evolved swarming-rate estimates higher than ancestral values (40, irrespective of individual-population significance levels), as opposed to unchanged or lower, is significantly greater than expected by chance (sign test, twotailed $P=0.001, N=55$ ), indicating that selection for numerical prevalence at the forefront of expanding swarms tends to result in faster swarming rates, but with many exceptions. When evolutionary swarming-rate changes were averaged across all populations within each of the six genotype-byenvironment treatments, mean cross-population swarming rate increased significantly in three treatments $(\mathrm{A}-\mathrm{S}+$ and $\mathrm{A}+\mathrm{S}-$ populations evolved on hard agar and $\mathrm{A}+\mathrm{S}+$ populations evolved on soft agar, Figure 2c).

It is clear that increased swarming rate is not the primary mode of competitive superiority for a large proportion of evolved populations. Among the 25 populations that did not swarm significantly faster than their ancestor, 13 swarming-rate estimates were actually lower than those of the respective ancestors, with three being significantly so (Figure $2 \mathrm{a}-\mathrm{b}$ and Supplementary Table S3).

Swarming-independent growth rates tended to increase but do not explain evolved swarming rates. We tested for generic, motility-independent adaptation to laboratory conditions by testing for increases in population growth rate that are manifested when growing populations are not allowed expand in territory size. Specifically, growth rates of evolved populations proliferating on the agar surfaces of their respective selection regimes without the opportunity for outward territory expansion were estimated. Within each treatment, the average growth rate across replicate populations increased significantly in five of the six treatments (Supplementary Figure S4). Across all populations, irrespective of environment, ten individual populations appear to have significantly increased their non-swarming growth rate (Table 1). These results indicate that, unsurprisingly, some degree of the overall adaptation achieved by evolved populations constitutes generic adaptation to laboratory conditions.

In theory, increased group-level swarming rates might have been caused solely by increases in motility-independent cell-division rate. In this scenario, faster swarm expansion would be driven simply by faster intrinsic population growth rather than by evolutionary changes in motility behavior. However, this does not appear to be the case, as no significant positive correlations between swarming rate and swarming-independent growth were detected among replicate populations (one-way analysis of covariance, $\mathrm{F}=1.9, \quad P=0.18$ ). Thus, evolutionary changes in swarming rate appear to have been often caused by changes in motility behavior per se.

Interaction with ancestral competitors stimulates faster swarming. In addition to evolving constitutively expressed increases in swarming rate, populations might have evolved to swarm faster specifically in presence of competitors. To test for this possibility, we compared the swarming rates of mixed swarms containing both evolved and ancestor cells (4 days after being initially mixed at a 1:1 ratio) to that of the respective evolved competitors alone. Averaged across all evolved populations, chimeric colonies composed of both ancestral and evolved genotypes swarmed significantly faster than evolved populations alone (Figure 3). This competition-specific 
a

Ancestor

$(\mathrm{A}+\mathrm{S}+)$

P3

P5

P8

P9

\section{P12}
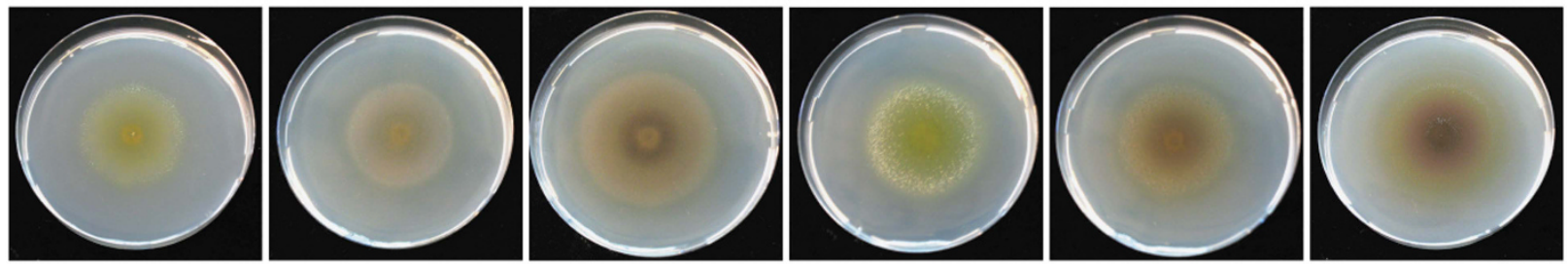

Ancestor

(A-S+)

P13

P15

P16

P17

P20
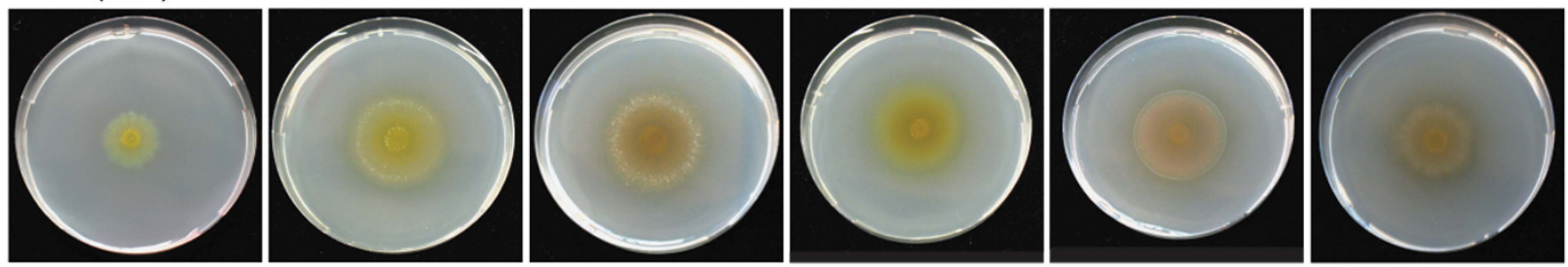

(A+S-)

P22

P24

P25

P26

P27
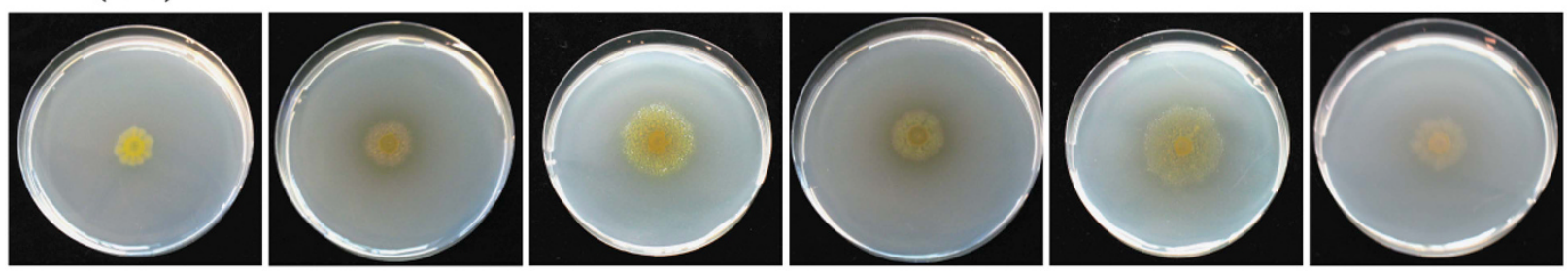

b

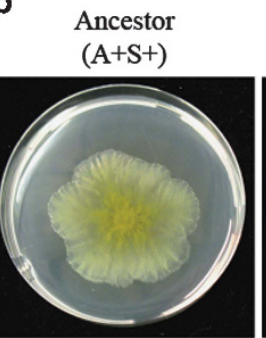

P30

P31

P34

P36

P37
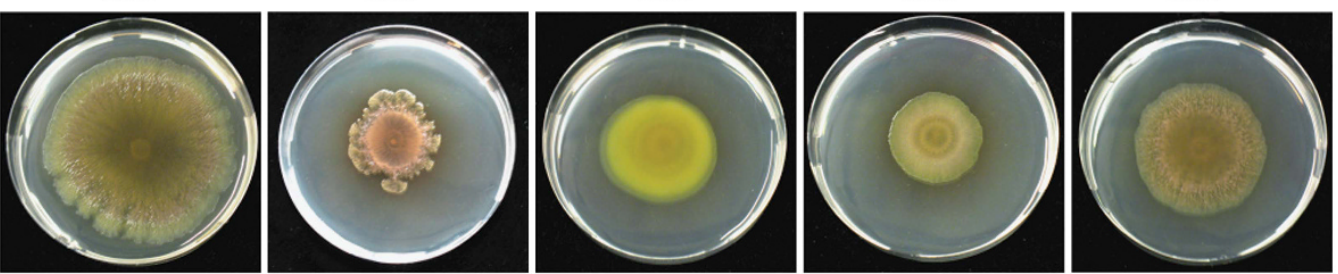

(A-S+)

P41

P42

P44

P45

P48

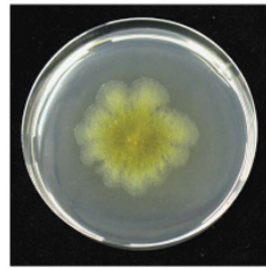

Ancestor

(A+S-)

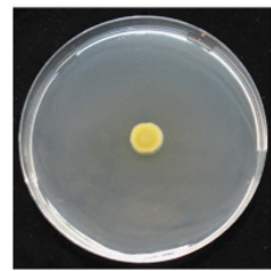

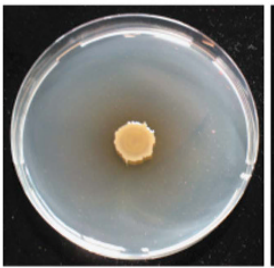

P49

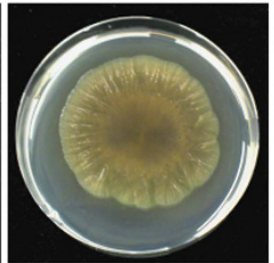

P50
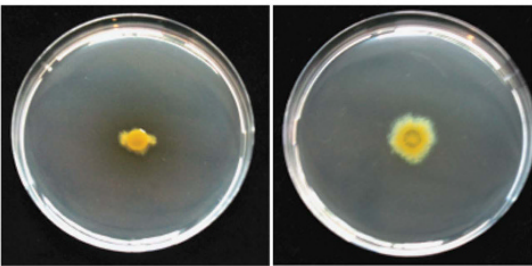

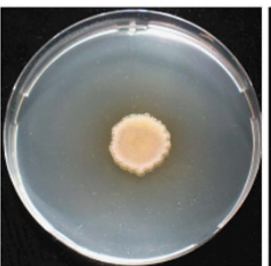

P52

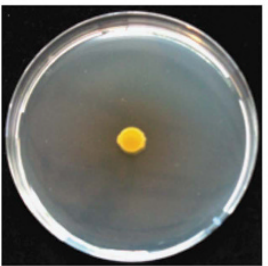

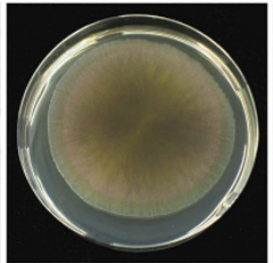

P55

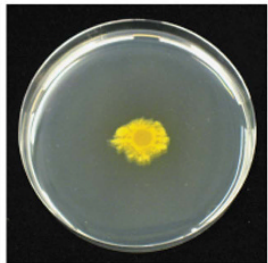

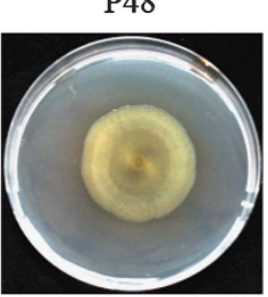

P56

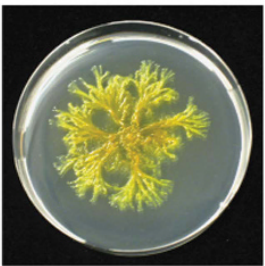

Figure 1 Swarm morphologies of evolved populations after 40 cycles. Swarming phenotypes after 7 days of growth by subsets of populations evolved on hard agar (a) and soft agar (b). 


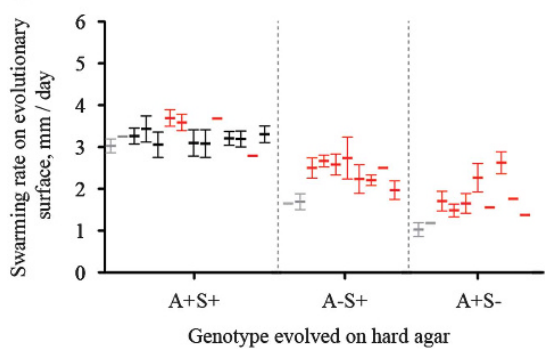

b

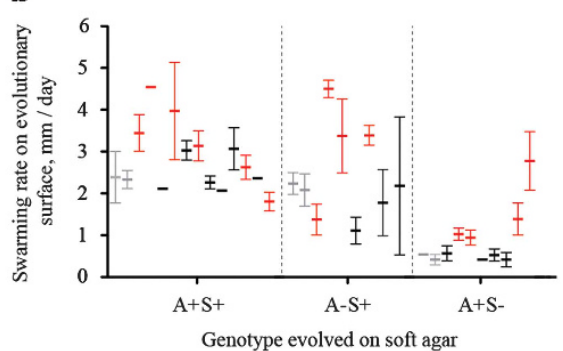

c

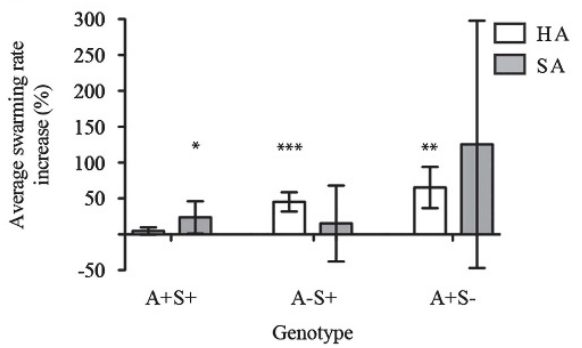

Figure 2 Swarming rates of evolved populations. Swarming rates of ancestors (gray) and each evolved population in its respective evolutionary environment, either hard agar (a) or soft agar (b). Evolved populations with a swarming rate that differed significantly from their respective ancestors are represented in red $(P<0.05)$, whereas populations with non-significant changes are in black. Swarming rates and $P$-values for each population can be found in Supplementary Table S3. Error bars represent 95\% confidence intervals (also for all other graphs unless indicated otherwise). (c) Average evolutionary change in swarming rates for each of the six treatments, expressed as a percentage increase relative to ancestor strains. ${ }^{*} P<0.05$; ${ }^{*} P<0.01 ;{ }^{*} * P<0.001$, asterisks indicate significant effect of evolutionary treatment on swarming rate as calculated by one-sample $t$-tests for differences from 0 .
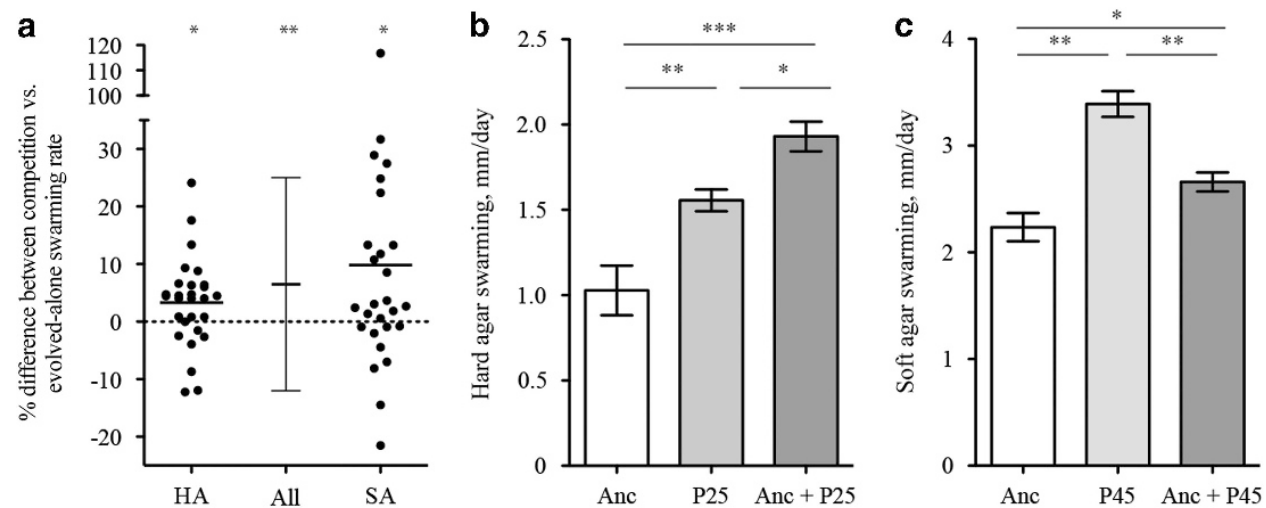

Figure 3 Interaction with ancestors affects evolved-population swarming rates. (a) Percentage differences between swarming rates of evolved populations in direct competition with their respective ancestor and evolved populations alone. Data points represent replicate-assay means for individual evolved populations. Horizontal lines indicate the average effect of evolved-ancestor mixing on evolved swarming rates across all populations evolved on each surface type and all populations in total. Significance levels correspond to Wilcoxon signed-rank tests for a difference from 0 and error bars represent standard deviations. (b) Mixed colonies of P25 and its ancestor swarm faster than either in pure culture. $P$-values reflect two-tailed paired $t$-tests between treatments connected by horizontal bars (also for c). (c) Mixed colonies of P45 and its ancestor swarm slower than $\mathrm{P} 45$ alone but faster than the ancestor alone. ${ }^{*} P<0.05 ;{ }^{*} P<0.01 ;{ }^{*} * P<0.001$.

increase in swarming rate was found to occur on both hard $(\sim 3.3 \%)$ and soft ( 13.2\%) agar (Figure 3). Such competitor-induced increases in swarming rate were significant for six individual populations (HAevolved P2, P18, P25 and SA-evolved P31, P32 and P38) (Figure $3 \mathrm{~b}$ and Table 1). Of these six populations, four swarm faster than their ancestors in pure culture (P2, P18, P25 and P32, Supplementary Table S3). Thus, in these cases, chimeric swarms were faster than either competitor alone. In the other two cases (P31 and P38), the evolved population alone was slower than the ancestral population alone (Supplementary Table S3) but swarmed faster in the presence of the ancestor. These results suggest that newly evolved abilities to increase swarming rate specifically in response to the presence of competitors may have contributed to adaptation in some populations.

Several populations evolved generic interference competition. Evolved interference-competition mechanisms might function generically across diverse environments (for example, a constitutively expressed anti-competitor compound) or might be specific to the leading edge of expanding swarms (for example, a motility-specific mechanism). We tested for generic interference-competition mechanisms by performing pairwise competition experiments during vegetative growth over the entire area of fencedin agar surfaces that allowed no outward swarm expansion. An indirect method to quantify the fitness of ancestors relative to their evolved competitors was used owing to strong cell-cell cohesion by $M$. xanthus during growth on surfaces (see Supplementary Material). After $24 \mathrm{~h}$ of growth by each mixed competition population on a fenced agar surface, the entire population was harvested and transferred to the center of a plate containing antibiotic that allowed growth only by the marked ancestor. The performance of each ancestor relative to its evolved competitor during the preceding competition stage was determined by comparing the relative sizes of ancestor colonies on selective plates that had been initiated from competitions 
against an evolved population vs those from control competitions against an unmarked ancestor. Using this method, five individual evolved populations (P13, P35, P49, P53 and P55) were found to significantly reduce ancestral growth during their respective competitions, with $\mathrm{P} 13$ and $\mathrm{P} 53$ having the strongest antagonistic effects (Supplementary Figure S5). Thus, several populations evolved interference competition that is effective in the absence of swarm expansion.

\section{Genotype and surface type effects on evolutionary}

\section{diversification}

Both ancestral genotype and selective environment can strongly influence the extent to which phenotypic traits evolve and such effects were evident among our populations. For example, populations that evolved on soft agar varied in swarming rate to a greater degree (relative to other populations derived from the same ancestor) in their selective regime than did populations that evolved on hard agar across all six genotypes (Figure 4a, $P=0.0035$, $N=6$ ). In particular, A-S+ populations swarming on soft agar evolved greater swarming-rate variance than any of the other five treatments. (Further analyses of genotype and environment effects on the degree of trait change are reported in Supplementary Information). In addition, genotype and environment might also influence the degree to which independent populations diversify from one another and we thus tested for such effects on diversification.

Variation in the absolute degree to which replicate populations evolve can be scaled to generate a parameter $-I_{X}$-that quantifies the extent of evolutionary diversification among populations that is independent of the mean degree of trait change (Vasi et al., 1994). More specifically, the diversification metric $I_{X}$ represents the variance of trait change among populations within a treatment divided by their mean trait change. This parameter can potentially be compared across evolutionary treatments to test for effects of known variables on trait diversification. In our experiments, mean $I_{X}$ values varied greatly across treatments (Table 2), suggesting strong effects of genotype and/or environment on evolutionary diversification.

To allow statistical comparison of $I_{X}$ across treatments, we calculated separate $I_{X}$ values for multiple pairs of populations within each treatment (always pairing populations that share the same ancestral marker state and without replacement) to generate multiple independent estimates of $I_{X}$ per treatment. This approach demonstrates that both genotype and environment (either alone or in combination) significantly affected the degree of evolutionary diversification across treatments, as mean $I_{X}$ differed significantly across several treatment pairs (Figure $4 \mathrm{~b}$ ). Interestingly, two treatments (A-S+ HA and A-S+ SA) sharing the same ancestral motility genotype (A-S+) exhibited the lowest and highest degrees of diversification (0.15 and 3.81, respectively), demonstrating that a difference in selective environments as simple as variable viscosity can greatly influence evolutionary diversification. Similarly, ancestral genotype also strongly affected diversification, as $\mathrm{A}+\mathrm{S}+$ populations evolving on hard agar diverged to a greater degree than did A-S+ populations.

\section{Evolutionary convergence in the frz and pil motility operons}

To begin investigating the molecular basis of adaptation and phenotypic evolution, we sequenced the genome of one representative clone from each of the 55 evolved populations examined here after 40 cycles of selection. The P29 clone was found to carry 435 mutations and is thus an apparent 'mutator' that was excluded from the following analyses. Among the other 54 clones, a total of 732 mutations were identified, giving an average of 14 mutations per clone. We first grouped all mutations by the Clusters of Orthologous Group (COG) process category to which the mutated genes belong (see Supplementary Table S4) (Tatusov et al., 2000)
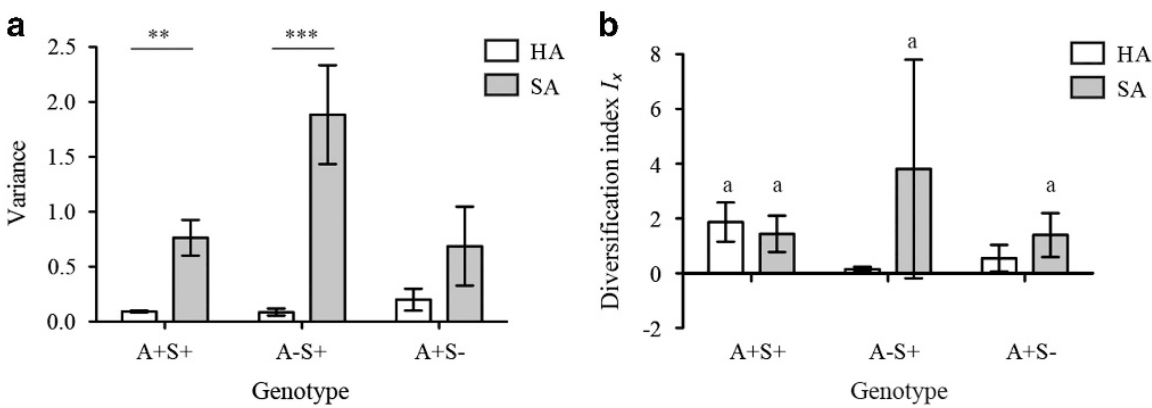

Figure 4 Diversification of evolved populations. (a) Within-treatment variance of swarming rates. $P$-values represent significance levels for differences between the hard and soft agar treatments for two genotypes after two-way ANOVA and Bonferroni post hoc tests. $N=12$ for $\mathrm{A}+\mathrm{S}+$ and $N=8$ for mutant genotypes. ${ }^{* *} P<0.01$; ${ }^{* *} P<0.001$. (b) Within-treatment diversification index $\left(I_{x}\right)$ for swarming rates. $I_{X}$ values here represent the average divergence among multiple independently evolved pairs of strains that each descended from the same ancestor and evolved in the same environment. 'a' represents $P<0.001$ for a difference between the respective treatment and the A-S+ hard agar treatment. 
Table 2 Comparison of diversification indices $\left(I_{x}\right)$ across independent experimental evolution studies

\begin{tabular}{|c|c|c|c|c|c|}
\hline Organism & Trait & Treatment & Generations & $I_{x}$ & Reference \\
\hline \multirow[t]{7}{*}{ M. xanthus } & Maximum growth rate & Growth in batch culture & 1000 & 0.23 & \multirow{7}{*}{$\begin{array}{l}\text { Velicer et al., } 1998 \\
\text { This study }\end{array}$} \\
\hline & Swarming rate & $\mathrm{A}+\mathrm{S}+$ on hard agar & $\geqslant 328$ & 1.70 & \\
\hline & & A-S+ on hard agar & $\geqslant 261$ & 0.35 & \\
\hline & & $A+S-$ on hard agar & $\geqslant 260$ & 0.58 & \\
\hline & & A+S+ on soft agar & $\geqslant 341$ & 1.64 & \\
\hline & & A-S+ on soft agar & $\geqslant 237$ & 2.72 & \\
\hline & & $\mathrm{A}+\mathrm{S}-$ on soft agar & $\geqslant 221$ & 1.60 & \\
\hline \multirow[t]{4}{*}{ P. aeruginosa } & Swarm area relative to ancestor & $\begin{array}{l}\text { Hard }(1.2 \%) \text { agar, selection for high } \\
\text { fitness at swarm edge }\end{array}$ & & 0.17 & \multirow[t]{4}{*}{$\begin{array}{l}\text { Taylor and } \\
\text { Buckling, } 2011\end{array}$} \\
\hline & & $\begin{array}{l}\text { Hard }(1.2 \%) \text { agar, spatially random } \\
\text { selection across swarm area }\end{array}$ & & 0.32 & \\
\hline & & $\begin{array}{l}\text { Soft }(0.3 \%) \text { agar, selection for high } \\
\text { fitness at swarm edge }\end{array}$ & & 0.15 & \\
\hline & & $\begin{array}{l}\text { Soft }(0.3 \%) \text { agar, spatially random } \\
\text { selection across swarm area }\end{array}$ & & 0.55 & \\
\hline \multirow[t]{8}{*}{ E. coli } & Relative fitness & Growth in batch culture & 2000 & 0.05 & \multirow[t]{8}{*}{ Vasi et al., 1994} \\
\hline & Lag period prior to growth & & 2000 & 0.29 & \\
\hline & Maximum growth rate & & 2000 & 0.06 & \\
\hline & Death rate & & 2000 & 0.57 & \\
\hline & Yield & & 2000 & 0.15 & \\
\hline & Death rate after long starvation & & 2000 & 0.34 & \\
\hline & Cell size—stationary phase & & 2000 & 0.21 & \\
\hline & Cell size-exponential phase & & 2000 & 0.19 & \\
\hline \multirow[t]{2}{*}{ Digital organisms } & Fitness & Simple environment & 4000 & $0.19(\max )$ & \multirow{2}{*}{$\begin{array}{l}\text { Zhang and } \\
\text { Travisano, } 2006\end{array}$} \\
\hline & & & 40000 & 0.05 (stable) & \\
\hline
\end{tabular}

and tested whether mutations were over- or underrepresented in each category. Whereas mutations in genes involved in carbohydrate and inorganic ion transport and metabolism (COG categories G and P), as well as in replication, recombination and repair (COG category L) were strongly under-represented (among other categories, Supplementary Table S4), mutations in genes involved in cell motility, signal transduction and intracellular trafficking, secretion and vesicular transport (COG categories $\mathrm{N}, \mathrm{T}$ and $\mathrm{U}$, respectively) were strongly over-represented (Supplementary Table S4).

Because a majority of populations exhibited significant increases in swarming rate, we further analyzed mutations in genes related to motility, the COG category that was most highly over-represented with mutations. We identified all mutations that occurred among genes previously known from experimental studies to affect $M$. xanthus motility (including two mutagenesis screens designed to identify all genes involved in both A and S motility (Youderian et al., 2003; Youderian and Hartzell, 2006)) and also examined mutations in several genes predicted by COG analysis to be motility-related but which have not yet been demonstrated experimentally to influence $M$. xanthus motility (Supplementary Table S5). In particular, we tested whether mutation patterns in the experimentally confirmed motility genes correlate with patterns of swarming-rate evolution.

Disproportionate numbers of mutations occurred in two multi-gene regions known to have critical roles in
M. xanthus motility, namely the frz and pil regions. The frz operon, which controls cell-reversal frequency in $M$. xanthus, was mutated in 42 populations. The frz operon encodes a complex chemosensory system, in which FrzCD acts as the chemoreceptor which is then methylated and demethylated by the methyltransferase FrzF and methylesterase FrzG, respectively. FrzCD activates the autophosphorylation of the histidine-kinase FrzE, which subsequently transfers the phosphoryl group to FrzZ, the response regulator that controls cell reversals during swarming which in turn controls swarm expansion (Kaimer et al., 2012). In addition, 11 different populations were mutated in the pil region, which is responsible for the production and function of the Type IV pili required for S motility (Wu and Kaiser, 1997) (Supplementary Table S5 and S6).

Mutations were found in only 15 of the $>100$ genes known from experimental studies to affect $M$. xanthus motility (for example, Sun et al., 2000; Caberoy et al., 2003; Youderian et al., 2003; Youderian and Hartzell, 2006; Kaimer et al., 2012): $\operatorname{agmK}(1$ mutation), frzCD (6 mutations), frzE (9), frzF (27), frzZ (3), mglA (1), mreB (1), mreC (1), nla19 (2), nla24 (4), pilA (5), pilB (1), pilG (3), pils (2) and pilT (1) (Supplementary Tables S4 and S5). A total of 67 mutations (9.1\% of all mutations) were found within these 15 genes, which together comprise only $0.2 \%$ of all annotated genes. Mutations in the frz and pil regions (8.3 and $21.6 \mathrm{~kb}$, respectively; 45 and 12 mutations, respectively) were extremely 
over-represented across all populations, indicating that they were major targets of selection, at least in many evolutionary treatments $\left(P<10^{-5}\right.$ for deviation from expectations of one and two mutations for the frz and pil regions, respectively, under a null assumption of random distribution of the 732 (non-P29) mutations across the $9.2 \mathrm{Mb} M$. xanthus genome, two-tailed binomial test).

Intriguingly, the frz and pil regions were not preferentially mutated specifically in those populations that exhibited significantly faster swarming than their ancestors. In fact, mutations in both operons were represented in a higher proportion of evolved populations with decreased swarming rates $(12 / 12(100 \%)$ and $4 / 12(33 \%)$ for frz and pil, respectively) than of populations with significantly increased rates $(20 / 31(65 \%)$ and 6/31 (19\%) for frz and pil, respectively). Under the plausible hypothesis that most mutations in these regions were adaptive, the lack of correlation between swarmingrate increases and the presence of mutations in the frz or pil regions raises intriguing questions regarding the effects of the mutations on fitness and behavior. For example, some frz or pil mutations may increase fitness and influence individual cell behavior without increasing overall swarming rate. Others may have increased swarming rate in the original genomic background in which they occurred only to have this effect dampened by subsequent adaptive mutations.
Owing to their numerical predominance, we examined mutations within the frz operon in greater detail. frz mutations were not distributed randomly within the operon. Three genes ( $f r z A, f r z B$ and $f r z G$ ) were not mutated in any population, whereas a disproportionately large number of mutations accumulated in the methyltransferase gene frzF (28 mutations (including P29), $P<0.001$ for deviation from the expectation of random distribution, binomial test). Within frzF (Figure 5), 17 out of 28 mutations preferentially localized to the SAMbinding domain, the functional domain that methylates the chemoreceptor FrzCD and triggers the signal transduction pathway. Mutation accumulation in this domain deviates significantly from the null expectation of a random distribution across the gene (binomial test, $P<0.001$ ).

Both environment and ancestral genotype affected patterns of mutation accumulation in the frz operon. A-S+ populations that evolved on hard agar (P13 to P20) constituted the only treatment that did not accumulate any frz mutations. In contrast, most A-S+ populations evolving on soft agar (five out of seven examined) did carry a frz mutation, indicating a strong environmental effect on the strength of selection on this operon for this motility genotype (Fisher's exact test, $P=0.007$ ). Ancestral genotype also influenced the types of $f r z$ mutation that accumulated. All frz mutations in $\mathrm{A}+\mathrm{S}+$ and $\mathrm{A}-\mathrm{S}+$ genotypes are non-synonymous single-nucleotide
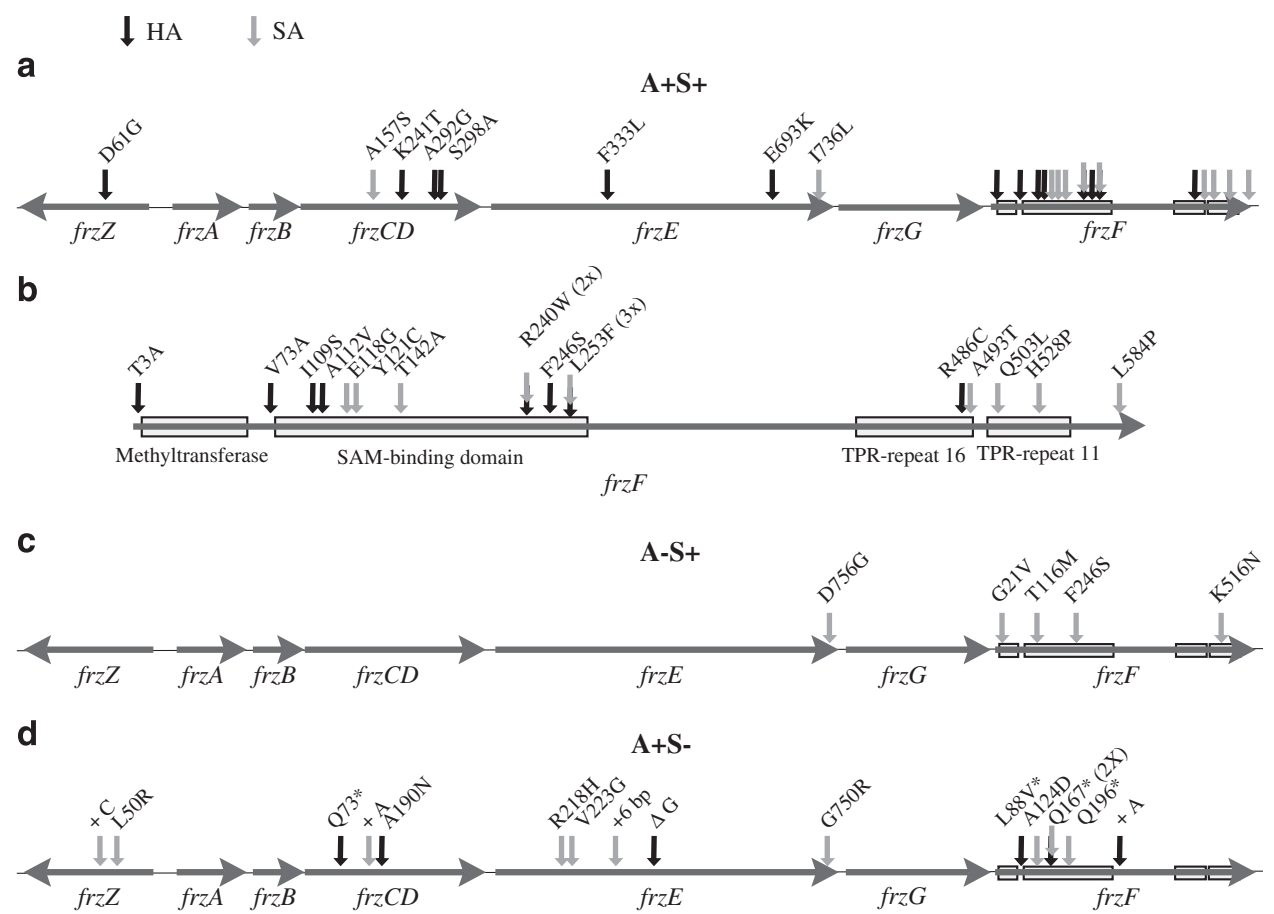

Figure 5 Selection on fitness at swarm fronts caused convergent evolution of the Frz chemosensory system in most treatments. Representations of the frz operon in M. xanthus. (a) frz mutations in A+S+ populations. Black and gray arrows indicate mutations in hardand soft-agar populations, respectively. Numbers indicate mutated amino-acid positions and letters indicate amino-acid changes. (b) Focus on the frzF gene highlighting regions encoding predicted functional domains as determined by Pfam (pfam.sanger.ac.uk/). (c, d) frz mutations in $\mathrm{A}-\mathrm{S}+$ and $\mathrm{A}+\mathrm{S}-$ populations, respectively. Asterisks indicate stop codons. 
polymorphisms that altered an amino acid in the encoded protein, whereas most mutations in $\mathrm{A}+\mathrm{S}-$ populations (10 out of 16) resulted in a frameshift or stop codon, suggesting loss of function.

\section{Discussion}

To investigate the mechanisms by which populations of motile microbes adapt when under selection to dominate the leading edge of an expanding colony, replicate populations of $M$. xanthus were allowed to evolve for a year and a half on two different surfaces. Previously published studies suggested that adaptation in such a selection regime may occur predominantly by simple increases in intrinsic motility rate that allow fast individuals to reach and disproportionately exploit unused growth substrates before their competitors arrive (Taylor and Buckling, 2011; Kearns, 2013; van Ditmarsch et al., 2013). In this study, however, patterns of trait change indicate that the dozens of $M$. xanthus populations examined here did not adapt merely by increasing swarming rate, but rather evolved a qualitatively diverse range of adaptations (Table 1).

A surprisingly large proportion of populations $(\sim 40 \%)$ greatly increased in fitness relative to their ancestor but did not exhibit a constitutively faster swarming rate, suggesting that some adapted by mechanisms also available to non-motile organisms. Indeed, populations tended to increase in their intrinsic growth rates even under conditions in which colonies were not allowed to expand in territory (Supplementary Figure S5), thus indicating that they adapted to general laboratory conditions to some degree. Several populations ( 10\%) evolved interference competition that hindered ancestral growth in non-swarming co-cultures (Table 1 and Supplementary Figure S5). Although antagonistic interactions are rampant among bacteria (Hibbing et al., 2010), little is known regarding the selective conditions that favor novel forms of allelopathy. Both theoretical and empirical studies have investigated the fitness effects of allelopathy as a function of passive dispersal (Chao and Levin, 1981; WlochSalamon et al., 2008) but how interference competition evolves in actively dispersing populations is not well understood. We found that interference competition can readily evolve even under selective conditions that often cause increases in the rate of active dispersal. Indeed, whereas two of the five populations that evolved interference competition did not swarm significantly faster than their ancestor, the three others did, including P13, which exhibited the most severe allelopathic effects on its ancestor. Further investigations into evolutionary relationships between interference competition and active dispersal are needed.

Unexpectedly, several individual populations evolved the ability to facultatively increase their swarming rate in response to the presence of competitors ( $10 \%$, Table 1 and Figure 3a). Moreover, there was a strong trend toward evolving such motility responsiveness to competitors across all populations (Figure 3). These results lend empirical credence to the possibility that microbes may frequently adapt by evolving the ability to facultatively alter social behaviors in response to variation in their social environment (Travisano and Velicer, 2004; Cornforth and Foster, 2013). In our populations, facultative alterations of motility rate might indeed have evolved as adaptations per se in their context of origin, but it is important to note that the hypothesis that these socially flexible behaviors may be pleiotropic byproducts of adaptive change at other traits cannot be excluded without further investigation (Rendueles et al., 2015). More generally, hypotheses that facultative responses by microbes to social or community interactions evolved as adaptations require careful testing.

For 14 evolved populations no significant differences from their ancestors were detected for any of the four traits we examined as possible targets of adaptation. Some populations may have in fact adapted via small changes in one or more of these traits that were not detected as being significant by our assays. Alternatively, these populations might have adapted by motility-specific mechanisms of interference competition. For example, such interference might be achieved by alteration of cellreversal patterns in a manner to which cells of the ancestral genotype respond dysfunctionally but which does not greatly alter the intrinsic swarming rate of an evolved population.

Our results also revealed that replicate populations within the same treatment diverged greatly in multiple respects, including (i) the direction of trait change (for example, the evolution of both slower and faster swarming in different populations, Table 1), (ii) the magnitude of trait change (for example, swarming rates of P41-P48, Figure 2a), (iii) the number of quantified traits other than fitness per se that changed significantly during evolution (ranging from zero to three, Table 1) and (iv) the specific patterns of significant trait changes (five and seven unique combinations among the $12 \mathrm{~A}+\mathrm{S}+\mathrm{HA}$ and $\mathrm{A}+\mathrm{S}+\mathrm{SA}$ populations, respectively, and either three or four unique combinations among the eight populations in each of the other four treatments).

To highlight one particular pattern of crossreplicate diversification, the $12 \mathrm{~A}+\mathrm{S}+$ populations that evolved on soft agar evolved nearly as much diversity in soft-agar swarming rates (relative to their average swarming rate) in 40 cycles (80 weeks) of selection as was found among 26 strains of $M$. xanthus that were isolated from a $16 \times 16 \mathrm{~cm}$ patch of soil (Vos and Velicer, 2008) ( $c \mathrm{~V}$ (coefficient of variation) $=0.29$ and 0.31 , respectively). The 12 $\mathrm{A}+\mathrm{S}+$ populations that were selected on hard agar also evolved a substantial fraction of the hard-agar swarming-rate diversity found among the same 26 
natural isolates ( $C V=0.08$ and 0.19 , respectively). Further, because the swarming-rate variation among our evolved populations that differ by few mutations is not much less than that observed among cm-scale natural isolates (Vos and Velicer, 2008) that differ genetically by tens of thousands of mutations (Wielgoss et al., 2016), it is clear that motility rate is a highly evolvable trait that can rapidly diversify across the range of rates maintained in the wild.

It is well established that differences in both genetic history (Travisano et al., 1995; Holder and Bull, 2001; Hall et al., 2010; Melnyk and Kassen, 2011; Vogwill et al., 2014) and environment (Travisano et al., 1995; MacLean and Bell, 2003; Melnyk and Kassen, 2011; Bailey and Kassen, 2012) can strongly influence patterns of evolutionary change at both phenotypic (Riley et al., 2001; Fong et al., 2005; Spor et al., 2014) and genetic levels (Dettman et al., 2012; Huang et al., 2014; Spor et al., 2014). These factors might also affect the degree to which independently evolving populations diversify (Korona et al., 1994; Tyerman et al., 2005; Melnyk and Kassen, 2011; Bailey and Kassen, 2012). In our experiments, this clearly was the case. Surface type affected the diversification level of $\mathrm{A}-\mathrm{S}+$ populations, whereas genotype (particularly $\mathrm{A}+\mathrm{S}+$ vs $\mathrm{A}-\mathrm{S}+$ ) affected the diversification level of populations evolving on hard agar (Figure 4).

In experiments with bacteria comparing adaptation during growth on agar surfaces versus in shaken liquid, Korona et al. (1994, 1996a, b), found that spatial structure promotes diversification relative to an unstructured habitat. Our results confirm the general principle that environment can influence diversification, but, in light of Korona et al. (1994), do so in an unexpected direction. Our evolutionary motility substrates, soft and hard agar, differed only in agar concentration, with soft agar being less structured owing to greater permeability. A-S+ populations that evolved on the less-structured surface diversified more than did those that evolved on hard agar. The directional contrast between our results and those of Korona et al., together with the fact that the surface-type treatment did not affect the relative degree of diversification among $\mathrm{A}+\mathrm{S}+$ or $\mathrm{A}+\mathrm{S}-$ populations, indicates that the relationship between degree of spatial structure and diversification can hinge on biological and environmental particularities.

Our results also show that ancestral genotypes differ in their propensity for trait diversification. Among the populations that evolved on hard agar, $\mathrm{A}+\mathrm{S}+$ populations diversified in swarming rate more than did either $\mathrm{A}-\mathrm{S}+$ or $\mathrm{A}+\mathrm{S}-$ populations. On soft agar, A-S+ populations diversified much more than did either $A$ $+\mathrm{S}+$ or $\mathrm{A}+\mathrm{S}-$ populations (Table 2 and Figure 4). Together, these results also illustrate that environment and genotype can interact to influence the degree of diversification among populations, as different ancestral genotypes show the greatest diversification across the two surface- type environments. Given that our ancestral motility genotypes differed pairwise by only one mutation, greater genetic differentiation might be expected to often influence diversification propensity to even greater degrees.

How does the degree of swarming-rate diversification documented here compare to diversification in other evolution experiments? Vasi et al. (1994) introduced the metric $I_{X}$ to quantify the degree of diversification at a trait of interest among independently evolved populations, scaled by the average degree of trait change. They calculated this parameter for fitness values among 12 populations of Escherichia coli that had undergone 2000 generations of growth in a liquid habitat, as well for several life-history traits that were potential targets of selection in that experiment (Vasi et al., 1994). Although heterogeneity was found in most traits and it was thus inferred that the E. coli populations followed somewhat different adaptive trajectories, the value of $I_{X}$ was well below unity for all traits (range of 0-0.57, Table 2), that is, the degree of interpopulation diversification was substantially lower than the average degree of trait change. Moreover, all traits that evolved did so in the same direction in all populations. In striking contrast, $I_{X}$ was much greater than unity in four of our six evolutionary treatments for measures of swarming rate on their respective selective-regime surface types (Table 2 ). In addition, in our experiments, the swarming rates of some populations derived from the same ancestor changed significantly in opposite directions in multiple treatments $(\mathrm{A}+\mathrm{S}+\mathrm{HA}, \mathrm{A}+\mathrm{S}+\mathrm{SA}$ and $\mathrm{A}-\mathrm{S}+\mathrm{SA})$.

The relative diversification of our populations is also much greater than that observed in a trait targeted by selection in 12 populations of $M$. xanthus after 1000 generations of evolution in liquid culture (Velicer et al., 1998). The value of $I_{X}$ for maximum growth rate in that study was 0.23 , which is lower than the smallest $I_{X}$ value reported here for swarming rate $\left(\mathrm{A}-\mathrm{S}+\mathrm{HA}, I_{X}=0.35\right)$. Diversification was also low in two previous studies in which swarming populations of Pseudomonas aeruginosa underwent evolution in selective regimes similar to ours (Taylor and Buckling, 2011; van Ditmarsch et al., 2013). Although extensive diversification has been previously observed in some evolution experiments (Rebolleda-Gomez et al., 2012), we are unaware of any set of replicate populations in which a quantifiable phenotypic trait has diversified more than the average degree of trait change (that is, had an $I_{x}$ value greater than unity), as occurred in four of our treatments. However, the useful diversification metric $I_{x}$ will need to be applied to many more evolution experiments in order to generate a frequency distribution of diversification ranges across organisms, traits, genotypes and environments before it can be inferred whether $I_{x}$ values above unity are rare or common across a much larger set of studies.

Convergent molecular evolution among replicate populations evolving in the same environment is common (Bull et al., 1997; Tenaillon et al., 2012; van 
Ditmarsch et al., 2013). Here, such convergence occurred strikingly at the frz operon (Figure 5, Supplementary Tables S5 and S6), which was mutated in all or a majority of replicate populations in all five treatments other than A-S+ HA (in which no frz mutations fixed in any population). Thus, both ancestral genotype and selective environment affected whether or not operon-level convergent evolution occurred. Intentionally introduced deletions or disruptions in the frz operon generally have negative effects on group-level swarming (Zusman et al., 2007; Kaimer et al., 2012). However, because most of our evolved populations swarm faster than their ancestors, one obvious hypothesis for how frz mutations might be adaptive in our selection regime is by increasing group-level swarming rate. This suggests that some mutations may enhance rather than reduce frz functions. In particular, some frzF mutations may change the methyl-group affinity of the encoded methyltransferase FrzF (which triggers the entire frz signal transduction cascade) in a manner that increases group-level swarming rate. Indeed, most of the mutations in FrzF occur in the methyl-binding site (or SAM-binding domain). However, how single point mutations in the frz operon may affect cell-reversal frequencies and group-level swarming remains unclear, as mutations in this operon were not statistically associated with increased swarming rates (or any other phenotype (Table 1)). frz-mutation benefits may often be contingent on social and/or genetic environments (for example, due to epistasis with other adaptive mutations (Chou et al., 2011; Kvitek and Sherlock, 2011; Angst and Hall, 2013)). For example, population-level behavioral effects caused by frz mutations in the genotypic context in which they were initially selected may have in many cases been masked by phenotypic changes caused by subsequent adaptive mutations in other genes. Because several populations carrying frz mutations do not swarm faster than their ancestors but nonetheless outcompete those ancestors (Table 1 and Supplementary Tables S2 and S3), some frz mutations may confer a fitness advantage not by enabling mutants to swarm faster, but rather by causing a motility-specific form of interference competition in which ancestral-genotype cells respond dysfunctionally to altered motility behavior by frz mutants.

Evolutionary increases in the proportion of overall fitness that is determined by social traits can be termed the 'socialization of fitness'. Fitness socialization should complexify adaptive landscape topographies (Mitri and Foster, 2013) because genes affecting social traits can increasingly interact epistatically across genomes (Wolf et al., 2000; Teseo et al., 2014) as well as within them (Phillips, 2008). The highly social character of myxobacterial life cycles (which often include social forms of motility, predation and multicellular development (Velicer and Vos, 2009)) combined with the unusually high levels of genetic complexity characteristic of myxobacterial genomes (Goldman et al., 2006) may together cause myxobacteria to be more evolutionarily diversifiable than less social prokaryotes. More broadly, the manners in which genetic variation can influence evolutionary diversification require further investigation.

Social environments can impose strong selective pressures. Because both forms of $M$. xanthus motility are social traits, early adaptive sweeps in our populations are likely to have fixed distinct mutations across replicate populations that differed in how they altered the social environments-and hence selective environments-of those populations. Such variation in the stochastic input of adaptive social mutations across populations may differentiate the subsequent set of potential adaptive trajectories open to those populations and thus promote diversification.

\section{Conflict of Interest}

The authors declare no conflict of interest.

\section{Acknowledgements}

We thank Sébastien Wielgoss and Jean-Claude Walser from the Genetic Diversity Center (GDC) at ETH Zürich for their help with bioinformatic analysis and SNP calling of evolved populations. We are grateful to Yuen-Tsu Nicco $\mathrm{Yu}$ and Dominique Schneider for helpful discussion and critical reading of the manuscript. We thank Francesca Fiegna and Michaela Amherd for technical assistance. OR is recipient of an EMBO (European Molecular Biology Organization) Long-Term Fellowship.

\section{References}

Angst DC, Hall AR. (2013). The cost of antibiotic resistance depends on evolutionary history in Escherichia coli. BMC Evol Biol 13.

Bailey SF, Kassen R. (2012). Spatial structure of ecological opportunity drives adaptation in a bacterium. Am Nat 180: 270-283.

Blount ZD, Borland CZ, Lenski RE. (2008). Historical contingency and the evolution of a key innovation in an experimental population of Escherichia coli. Proc Natl Acad Sci USA 105: 7899-7906.

Bowler DE, Benton TG. (2005). Causes and consequences of animal dispersal strategies: relating individual behaviour to spatial dynamics. Biol Rev C Phil Soc 80: $205-225$.

Bull JJ, Badgett MR, Wichman HA, Huelsenbeck JP, Hillis DM, Gulati A et al. (1997). Exceptional convergent evolution in a virus. Genetics 147: 1497-1507.

Caberoy NB, Welch RD, Jakobsen JS, Slater SC, Garza AG. (2003). Global mutational analysis of NtrC-like activators in Myхососcus xanthus: identifying activator mutants defective for motility and fruiting body development. J Bacteriol 185: 6083-6094.

Chao L, Levin BR. (1981). Structured habitats and the evolution of anticompetitor toxins in bacteria. Proc Natl Acad Sci USA 78: 6324-6328. 
Chou HH, Chiu HC, Delaney NF, Segre D, Marx CJ. (2011). Diminishing returns epistasis among beneficial mutations decelerates adaptation. Science 332: 1190-1192.

Cornforth DM, Foster KR. (2013). Competition sensing: the social side of bacterial stress responses. Nat Rev Microbiol 11: 285-293.

Dettman JR, Rodrigue N, Melnyk AH, Wong A, Bailey SF, Kassen R. (2012). Evolutionary insight from wholegenome sequencing of experimentally evolved microbes. Mol Ecol 21: 2058-2077.

Fong SS, Joyce AR, Palsson BO. (2005). Parallel adaptive evolution cultures of Escherichia coli lead to convergent growth phenotypes with different gene expression states. Genome Res 15: 1365-1372.

Goldman BS, Nierman WC, Kaiser D et al. (2006). Evolution of sensory complexity recorded in a myxobacterial genome. Proc Natl Acad Sci USA 103: 15200-15205.

Hall AR, Griffiths VF, MacLean RC, Colegrave N. (2010). Mutational neighbourhood and mutation supply rate constrain adaptation in Pseudomonas aeruginosa. Proc Bio Sci R Soc 277: 643-650.

Hibbing ME, Fuqua C, Parsek MR, Peterson SB. (2010). Bacterial competition: surviving and thriving in the microbial jungle. Nat Rev Microbiol 8: 15-25.

Hillesland KL, Velicer GJ. (2005). Resource level affects relative performance of the two motility systems of Myxococcus xanthus. Microb Ecol 49: 558-566.

Hodgkin J, Kaiser D. (1979). Genetics of gliding motility on Myxococcus xanthus (myxobacteriales)-two gene systems control movement. Mol Gen Genet 171: 177-191.

Holder KK, Bull JJ. (2001). Profiles of adaptation in two similar viruses. Genetics 159: 1393-1404.

Huang Y, Wright SI, Agrawal AF. (2014). Genome-wide patterns of genetic variation within and among alternative selective regimes. PLoS Genet 10: e1004527.

Josenhans C, Suerbaum S. (2002). The role of motility as a virulence factor in bacteria. Int J Med Microbiol 291: 605-614.

Kaimer C, Berleman JE, Zusman DR. (2012). Chemosensory signaling controls motility and subcellular polarity in Myxococcus xanthus. Curr Opin Microbiol 15: 751-757.

Kaiser D. (1979). Social gliding is correlated with the presence of pili in Myxococcus xanthus. Proc Natl Acad Sci USA 76: 5952-5956.

Kawecki TJ, Lenski RE, Ebert D, Hollis B, Olivieri I, Whitlock MC. (2012). Experimental evolution. Trends Ecol Evol 27: 547-560.

Kearns DB. (2013). You get what you select for: better swarming through more flagella. Trends Microbiol 21: $508-509$.

Korona R. (1996a). Adaptation to structurally different environments. P Roy Soc B Biol Sci 263: 1665-1669.

Korona R. (1996b). Genetic divergence and fitness convergence under uniform selection in experimental populations of bacteria. Genetics 143: 637-644.

Korona R, Nakatsu CH, Forney LJ, Lenski RE. (1994). Evidence for multiple adaptive peaks from populations of bacteria evolving in a structured habitat. Proc Natl Acad Sci USA 91: 9037-9041.

Kraemer SA, Velicer GJ. (2011). Endemic social diversity within natural kin groups of a cooperative bacterium. Proc Natl Acad Sci USA 108: 10823-10830.

Kvitek DJ, Sherlock G. (2011). Reciprocal sign epistasis between frequently experimentally evolved adaptive mutations causes a rugged fitness landscape. PLoS Genet 7: e1002056.
Lemonnier M, Levin BR, Romeo T, Garner K, Baquero MR, Mercante J et al. (2008). The evolution of contactdependent inhibition in non-growing populations of Escherichia coli. Proc Bio Sci R Soc 275: 3-10.

MacLean RC, Bell G. (2003). Divergent evolution during an experimental adaptive radiation. Proc Bio Sci $R$ Soc 270: $1645-1650$.

Magsig-Castillo J, Morse JG, Walker GP, Bi JL, Rugman-Jones PF, Stouthamer R. (2010). Phoretic dispersal of armored scale crawlers (Hemiptera: Diaspididae). J Econ Entom 103: 1172-1179.

Melnyk AH, Kassen R. (2011). Adaptive landscapes in evolving populations of Pseudomonas fluorescens. Evolution 65: 3048-3059.

Mignot T, Shaevitz JW, Hartzell PL, Zusman DR. (2007). Evidence that focal adhesion complexes power bacterial gliding motility. Science 315: 853-856.

Mitri S, Foster KR. (2013). The genotypic view of social interactions in microbial communities. Annu Rev Genet 47: 247-273.

Ottemann KM, Miller JF. (1997). Roles for motility in bacterial-host interactions. Mol Microbiol 24: 1109-1117.

Phillips PC. (2008). Epistasis-the essential role of gene interactions in the structure and evolution of genetic systems. Nat Rev Genet 9: 855-867.

Pratt LA, Kolter R. (1998). Genetic analysis of Escherichia coli biofilm formation: roles of flagella, motility, chemotaxis and type I pili. Mol Microbiol 30: 285-293.

Rebolleda-Gomez M, Ratcliff W, Travisano M. (2012). Adaptation and divergence during experimental evolution of multicellular Saccharomyces cerevisiae. Artif Life 13: 99-104.

Rendueles O, Zee PC, Dinkelacker I, Amherd M, Wielgoss S, Velicer GJ. (2015). Rapid and widespread de novo evolution of kin discrimination. Proc Natl Acad Sci USA 112: 9076-9081.

Reynolds AM, Bohan DA, Bell JR. (2006). Ballooning dispersal in arthropod taxa with convergent behaviours: dynamic properties of ballooning silk in turbulent flows. Biol Lett 2: 371-373.

Riley MS, Cooper VS, Lenski RE, Forney LJ, Marsh TL. (2001). Rapid phenotypic change and diversification of a soil bacterium during 1000 generations of experimental evolution. Microbiology 147: 995-1006.

Rodriguez AM, Spormann AM. (1999). Genetic and molecular analysis of $\operatorname{cglB}$, a gene essential for singlecell gliding in Myxococcus xanthus. J Bacteriol 181: $4381-4390$.

Shi W, Zusman DR. (1993). The two motility systems of Myxococcus xanthus show different selective advantages on various surfaces. Proc Natl Acad Sci USA 90: 3378-3382.

Spor A, Kvitek DJ, Nidelet T, Martin J, Legrand J, Dillmann C et al. (2014). Phenotypic and genotypic convergences are influenced by historical contingency and environment in yeast. Evolution 68: 772-790.

Stocker R, Seymour JR, Samadani A, Hunt DE, Polz MF. (2008). Rapid chemotactic response enables marine bacteria to exploit ephemeral microscale nutrient patches. Proc Natl Acad Sci USA 105: 4209-4214.

Sun H, Zusman DR, Shi W. (2000). Type IV pilus of Myxococcus xanthus is a motility apparatus controlled by the frz chemosensory system. Curr Biol 10: 1143-1146. 
Sun M, Wartel M, Cascales E, Shaevitz JW, Mignot T. (2011). Motor-driven intracellular transport powers bacterial gliding motility. Proc Natl Acad Sci USA 108: $7559-7564$.

Swingland IR, Greenwood PJ. (1983). The Ecology of Animal Movement. Oxford University Press: New York, NY, USA.

Tatusov RL, Galperin MY, Natale DA, Koonin EV. (2000). The COG database: a tool for genome-scale analysis of protein functions and evolution. NAR 28: 33-36.

Taylor JR, Stocker R. (2012). Trade-offs of chemotactic foraging in turbulent water. Science 338: 675-679.

Taylor TB, Buckling A. (2011). Selection experiments reveal trade-offs between swimming and twitching motilities in Pseudomonas aeruginosa. Evolution 65: 3060-3069.

Taylor TB, Buckling A. (2013). Bacterial motility confers fitness advantage in the presence of phages. J Evol Biol 26: 2154-2160.

Tenaillon O, Rodriguez-Verdugo A, Gaut RL, McDonald P, Bennett AF, Long AD et al. (2012). The molecular diversity of adaptive convergence. Science 335: $457-461$.

Teseo S, Chaline N, Jaisson P, Kronauer DJ. (2014). Epistasis between adults and larvae underlies caste fate and fitness in a clonal ant. Nat Commun 5: 3363.

Travisano M, Velicer GJ. (2004). Strategies of microbial cheater control. Trends Microbiol 12: 72-78.

Travisano M, Mongold JA, Bennett AF, Lenski RE. (1995). Experimental tests of the roles of adaptation, chance, and history in evolution. Science 267: 87-90.

Turnbull GA, Morgan JA, Whipps JM, Saunders JR. (2001). The role of bacterial motility in the survival and spread of Pseudomonas fluorescens in soil and in the attachment and colonisation of wheat roots. FEMS Microbiol Ecol 36: 21-31.

Tyerman J, Havard N, Saxer G, Travisano M, Doebeli M. (2005). Unparallel diversification in bacterial microcosms. Proc Bio Sci R Soc 272: 1393-1398.

van Ditmarsch D, Boyle KE, Sakhtah H, Oyler JE, Nadell CD, Deziel E et al. (2013). Convergent evolution of hyperswarming leads to impaired biofilm formation in pathogenic bacteria. Cell Rep 4: 697-708.

Vasi F, Travisano M, Lenski RE. (1994). Long-term experimental evolution in Escherichia coli 2. Changes in life-history traits during adaptation to a seasonal environment. Am Nat 144: 432-456.

Velicer GJ, Yu YT. (2003). Evolution of novel cooperative swarming in the bacterium Myxococcus xanthus. Nature 425: 75-78.

Velicer GJ, Vos M. (2009). Sociobiology of the myxobacteria. Annu Rev Microbiol 63: 599-623.

Velicer GJ, Kroos L, Lenski RE. (1998). Loss of social behaviors by Myхососcus xanthus during evolution in an unstructured habitat. Proc Natl Acad Sci USA 95: 12376-12380.
Vogwill T, Kojadinovic M, Furio V, MacLean RC. (2014). Testing the role of genetic background in parallel evolution Using the comparative experimental evolution of antibiotic resistance. Mol Biol Evol 31: 33143323.

Vos M, Velicer GJ. (2008). Natural variation of gliding motility in a centimetre-scale population of Myxococcus xanthus. FEMS Microbiol Ecol 64: 343-350.

Wielgoss S, Didelot X, Chaudhuri RR, Liu X, Weedall GD, Velicer GJ et al. (2016). A barrier to homologous recombination between sympatric strains of the cooperative soil bacterium Myxococcus xanthus. ISME J; e-pub ahead of print 5 April 2016; doi:10.1038/ismej.2016.34.

Wloch-Salamon DM, Gerla D, Hoekstra RF, de Visser JA. (2008). Effect of dispersal and nutrient availability on the competitive ability of toxin-producing yeast. Proc Bio Sci R Soc 275: 535-541.

Wolf JB, Brodie ED, M.J. W. (2000). Epistasis and the evolutionary process. Oxford University Press: New York, NY, USA.

Wu SS, Kaiser D. (1995). Genetic and functional evidence that Type IV pili are required for social gliding motility in Myxococcus xanthus. Mol Microbiol 18: 547-558.

Wu SS, Kaiser D. (1997). Regulation of expression of the pilA gene in Myxococcus xanthus. J Bacteriol 179: 7748-7758.

Youderian P, Hartzell PL. (2006). Transposon insertions of magellan-4 that impair social gliding motility in Myxососcus xanthus. Genetics 172: 1397-1410.

Youderian P, Burke N, White DJ, Hartzell PL. (2003). Identification of genes required for adventurous gliding motility in Myxococcus xanthus with the transposable element mariner. Mol Microbiol 49: $555-570$.

Young IM, Crawford JW. (2004). Interactions and selforganization in the soil-microbe complex. Science 304: 1634-1637.

Zhang H, Travisano M. (2006). Predicting fitness effects of beneficial mutations in digital organisms. Ieee Symp Art Life, 39-46.

Zusman DR, Scott AE, Yang Z, Kirby JR. (2007). Chemosensory pathways, motility and development in Myxococcus xanthus. Nat Rev Microbiol 5: 862-872.

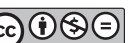

This work is licensed under a Creative Commons Attribution-NonCommercialNoDerivs 4.0 International License. The images or other third party material in this article are included in the article's Creative Commons license, unless indicated otherwise in the credit line; if the material is not included under the Creative Commons license, users will need to obtain permission from the license holder to reproduce the material. To view a copy of this license, visit http://creativecommons.org/ licenses/by-nc-nd/4.0/

Supplementary Information accompanies this paper on The ISME Journal website (http://www.nature.com/ismej) 